\title{
Topological phases in small quantum Hall samples
}

\author{
Tobias Graß, ${ }^{1}$ Bruno Juliá-Díaz, ${ }^{1,2}$ and Maciej Lewenstein ${ }^{1,3}$ \\ ${ }^{1}$ ICFO-Institut de Ciències Fotòniques, Parc Mediterrani de la Tecnologia, 08860 Barcelona, Spain \\ ${ }^{2}$ Departament d'Estructura i Constituents de la Matèria, Universitat de Barcelona, 08028 Barcelona, Spain \\ ${ }^{3}$ ICREA-Institució Catalana de Recerca i Estudis Avançats, 08010 Barcelona, Spain
}

(Received 14 October 2013; published 27 January 2014)

\begin{abstract}
Topological order has proven a useful concept to describe quantum phase transitions which are not captured by the Ginzburg-Landau type of symmetry-breaking order. However, lacking a local order parameter, topological order is hard to detect. One way to detect it is via direct observation of anyonic properties of excitations which are usually discussed in the thermodynamic limit, but so far has not been realized in macroscopic quantum Hall samples. Here we consider a system of few interacting bosons subjected to the lowest Landau level by a gauge potential, and theoretically investigate vortex excitations in order to identify topological properties of different ground states. Our investigation demonstrates that even in surprisingly small systems anyonic properties are able to characterize the topological order. In addition, focusing on a system in the Laughlin state, we study the robustness of its anyonic behavior in the presence of tunable finite-range interactions acting as a perturbation. A clear signal of a transition to a different state is reflected by the system's anyonic properties.
\end{abstract}

DOI: 10.1103/PhysRevA.89.013623

PACS number(s): 67.85.De, 73.43.-f

\section{INTRODUCTION}

The discovery of the quantum Hall effects [1-3] has ushered a new era of quantum many-body physics: Without undergoing the Ginzburg-Landau mechanism of spontaneous symmetry breaking, a quantum phase transition takes place whenever a quantum Hall system jumps from one to another Hall plateau upon tuning the external magnetic field strength. This phenomenology has been described in terms of an effective topological quantum field theory $[4,5]$, giving rise to the concepts of "topological quantum numbers" and "topological order" which allows one to distinguish between Hall plateaus. However, this new form of order cannot simply be described by a local order parameter. In fact, it is a nonlocal property stemming from the highly correlated nature of topologically ordered states, and thus robust against local perturbations. On the one hand, this makes topological quantum matter an interesting environment for realizing topologically protected qubits, topologically protected quantum memory, and performing quantum computations [6]. On the other hand, the measurement of topological order is an intricate issue [7-9].

From the theoretical point of view, progress has been made in classifying different types of topological matter without topological order, such as noninteracting topological insulators of fermions [10,11], or symmetry-protected topological phases of interacting bosons $[12,13]$. For characterizing topological order, different theoretical tools have been established: It has first been argued that topological order manifests itself when the quantum state is put onto a topologically nontrivial surface such as a torus [5]. Depending on the genus of the surface, there is a different number of ways how particles can arrange themselves in accordance with the long-range entanglement dictated by the topological order. This gives rise to a characteristic number of degenerate ground states. In topologically trivial geometries, such as a disk or a sphere, this classification amounts to counting the number of degenerate edge states [5,14]. Importantly, the number of edge states equals the number of low-lying states in the spectrum of the reduced density matrix $[15,16]$. This allows for analyzing the topological order via edge-state counting even in the absence of physical edges (e.g., on a sphere). In all cases, however, finite-range interactions and the finite size of the system may lift the characteristic degeneracies, without necessarily destroying the topological order.

The von Neuman entropy has been discussed as another criterion to characterize topological order $[17,18]$. It has been argued that, for a topologically ordered state, it contains, in addition to the usual part which scales with the area of the system, a constant part characteristic of the topological order. This criterion has successfully been applied to quantum Hall states [19-21], but it requires the measurement of entropies for differently sized systems in order to extract the relevant information.

The most natural and direct manifestation of topological order seems to be the anyonic properties of the excitations, that is, the fractional [22] or even non-Abelian statistics [23] of quasiparticle or quasihole excitations. Apart from being a signature of the topological order, anyons are interesting on their own, as they do not match with the usual classification of particles into bosons or fermions. Three decades after their theoretical prediction [3,22], anyons have remained rather exotic quasiparticles: A clear experimental detection has so far only been achieved with respect to their fractional charge $[24,25]$, but not with respect to the fractional statistics. The latter has been proposed based on interference effects during the edge-to-edge tunneling of electrons [26], however, experiments so far $[27,28]$ have not been carried out in the fractional quantum Hall regime.

The difficulties in handling anyons in solid-state systems motivate the implementation of quantum Hall Hamiltonians in systems which offer a large amount of control. In the past decade, a great deal of attention has been paid to quantum gases. Though the atoms' electroneutrality hinders an implementation of quantum Hall physics by real magnetic fields, several ways of achieving artificial magnetic fields have been discussed and realized. Conceptually, the simplest of them is a rotation of the atomic cloud by which a Coriolis force 
mimics the Lorentz force on a charged particle in a magnetic field [29-31]. Other schemes consider the generation of laserinduced Berry phases [32-38], or Berry phases induced by shaking of an optical lattice [39]. Provided with such artificial gauge fields, cold atoms should support different quantum Hall states, if the gauge field is sufficiently strong to bring all particles into the lowest Landau level [40-45]. In particular, repulsive two-body contact interactions in a single component bosonic cloud ideally support the Laughlin state at filling factor $v=1 / 2$ [or alternatively at total angular momentum $L$ in $z$ direction given by $L=\hbar N(N-1)$ ] as an exact solution. With a laser focused onto the atomic cloud, one can then produce localized excitations [46-48]. Their anyonic properties can be studied by adiabatically moving the potential. As explicitly shown in Ref. [48] by analysis of the Laughlin wave function, even for systems as small as $N \gtrsim 5$ there is a bulk in the atomic cloud which is broad enough to pierce two quasiholes sufficiently far from one another and from the edge of the system, such that the statistical phase takes the value $1 / 2$, expected in the thermodynamic limit from the plasma analogy $[5,22]$. That is, at least for the case of the Laughlin state, topological order manifests itself even in very small systems in the form of anyons. Apart from the cold atomic systems envisaged in this paper, mesoscopic quantum Hall samples can also be thought of as photons in artificial gauge potentials [49], or quantum dot electrons in a magnetic field (cf. Refs. [50-52], and references therein).

In this paper, we first apply the program of Ref. [48] to a larger class of states with topological order, in particular to the 221-Halperin state, the ground state of two-component bosons with two-body contact repulsion, the $v=1 / 4$ Laughlin state, and composite fermion states [53]. We find that the fractional charge of a vortex is related to the filling fraction of these states in the thermodynamic limit, but accessible system sizes are too small for a clear signature of the fractional statistics. In the second part of this paper, we focus on the $v=1 / 2$ Laughlin state, as the one with the clearest anyonic signatures at small system size. We investigate how its anyonic properties are modified when the system is perturbed by some rotationally symmetric, finite-range interactions, keeping fixed the total angular momentum of the system. In this way, our study is complementary to the one presented in Ref. [48], where we investigated the role of a symmetry-breaking perturbation at the single-particle level. In the latter case, the ground state is to a large extent a superposition of the Laughlin state plus several edge excitations, so the robustness of anyonic properties does not come as a surprise.

The rotationally symmetric scenario considered here directly relates to the very general situation where the topological trial state is not an exact solution for a given interaction, as is the case in the "original" fractional quantum Hall system of electrons with Coulomb interactions, or in the case of atoms with dipolar interactions. A common scenario is also the one where atoms interact with two-body contact interaction, but the total angular momentum is too small to support the Laughlin state, giving rise to large overlaps $\lesssim 1$ with topological states from the Read-Rezayi series [41,54], or the non-Abelian spin singlet (NASS) series [44,45,55]. Usually, in such cases it is argued that a sizable overlap of the exact ground state with the trial state for a small number of particles indicates that the system is in the same topological phase. This argumentation is often backed by considering other criteria for topological order, such as the above-mentioned ground state degeneracy on a torus geometry, which is not unique unless one reaches the thermodynamic limit. In the example studied in this paper, we show that the topological order of a perturbed Laughlin state can be directly seen from the anyonic properties of the vortex excitations. This suggests that, despite the smallness of the considered system $(N=6)$, a topological phase is spanned within the finite Hilbert space, in contrast to a single, topologically ordered state.

\section{THE SYSTEM}

We consider a two-dimensional system of bosonic atoms with mass $M$, described by the effective Hamiltonian $\mathcal{H}=$ $\sum_{i=1}^{N} H_{i}+\mathcal{V}$, where the single-particle contribution reads

$$
H_{i}=\frac{\left(\boldsymbol{p}_{i}+\boldsymbol{A}_{i}\right)^{2}}{2 M}+\frac{M}{2} \omega_{\mathrm{eff}}^{2} r_{i}^{2} .
$$

The kinetic term is coupled to a gauge potential $\boldsymbol{A}_{i}$, acting on the $i$ th particle, and for convenience, we choose the symmetric gauge, $\boldsymbol{A}_{i}=\frac{B}{2}\left(y_{i},-x_{i}, 0\right)$, with $B$ the gauge field strength. Different proposals for synthesizing such gauge potential for atoms are reviewed in Refs. [30,33]. This first term of the Hamiltonian thus gives rise to a Landau level (LL) structure, with equidistant energy levels. The second term is an effective trapping potential with frequency $\omega_{\text {eff }}$. It can be used to control the $z$ component of total angular momentum of the system. For simplicity, we define $\hbar \omega \equiv \sqrt{\omega_{\text {eff }}^{2}+\frac{B^{2}}{4 M^{2}}}$ as a unit of energy, and $\lambda=\sqrt{\hbar /(M \omega)}$ as a unit of length. The LL gap, $\Delta_{\mathrm{LL}}=$ $2 \eta$, and the degeneracy splitting of each LL due to the trap, $\delta \equiv 1-\eta$, are then expressed in terms of the dimensionless parameter $\eta \equiv B /(2 M \omega) \leqslant 1$.

We first consider the spinless case (bosons of only one species), and assume that the system can be restricted to the lowest LL (LLL). The LL energy spectrum is given by $E_{\ell}=\delta \ell+$ constant, corresponding to the Fock-Darwin (FD) states

$$
\phi_{\ell}^{\mathrm{FD}}(z)=\frac{1}{\sqrt{2 \pi 2^{\ell} \ell !}} z^{\ell} \exp \left(-|z|^{2} / 4\right),
$$

with $z=x+i y$, and $\ell$ the single-particle $z$ component of the angular momentum. The many-body ground state in the noninteracting system, $\mathcal{V}=0$, will be a condensate wave function at zero angular momentum, $\ell=0$, given by

$$
\Psi_{0}\left(z_{1}, \ldots, z_{N}\right)=\prod_{i=1}^{N} \phi_{0}^{\mathrm{FD}}\left(z_{i}\right) .
$$

Repulsive interactions may bring the system into different strongly correlated, topological states, depending on the range of the interactions and the ratio between interaction strength and effective trapping frequency. A realistic model for the interactions in a Bose gas is repulsive $s$-wave scattering, which in a many-body notation reads

$$
\mathcal{V}_{0}=V_{0} \sum_{\ell_{1}, \ldots, \ell_{4}} v_{\ell_{1}, \ell_{2}, \ell_{3}, \ell_{4}} a_{\ell_{1}}^{\dagger} a_{\ell_{2}}^{\dagger} a_{\ell_{3}} a_{\ell_{4}},
$$


where $V_{0}$ parametrizes the interaction strength, and $a_{\ell}$ $\left(a_{\ell}^{\dagger}\right)$ are the annihilation (creation) operators for a boson in state $\phi_{\ell}^{\mathrm{FD}}$. The matrix elements $v_{\ell_{1}, \ell_{2}, \ell_{3}, \ell_{4}}$ are given by $\left\langle\ell_{1}, \ell_{2}\left|\delta\left(z-z^{\prime}\right)\right| \ell_{3}, \ell_{4}\right\rangle \sim \delta_{\ell_{1}+\ell_{2}, \ell_{3}+\ell_{4}}$. Other interactions, with nonzero range, are conveniently expressed in terms of Haldane pseudopotentials. Therefore, one changes from a basis $\left|\ell_{1}, \ell_{2}\right\rangle \equiv \phi_{\ell_{1}}^{\mathrm{FD}}\left(z_{1}\right) \phi_{\ell_{2}}^{\mathrm{FD}}\left(z_{2}\right)$ into a basis $|n, l\rangle \equiv \phi_{n}^{\mathrm{FD}}(Z) \phi_{l}^{\mathrm{FD}}(z)$ with the center-of-mass coordinate $Z=\left(z_{1}+z_{2}\right) / \sqrt{2}$, and the relative coordinate $z=\left(z_{1}-z_{2}\right) / \sqrt{2}$. We straightforwardly find $\left|\ell_{1} \ell_{2}\right\rangle=\sum_{n, l} C_{\ell_{1}, \ell_{2}}^{n, l}|n, l\rangle$ with

$$
\begin{aligned}
C_{\ell_{1}, \ell_{2}}^{n, l}= & \delta_{\ell_{1}+\ell_{2}, n+l} \sqrt{\frac{\ell_{1} ! \ell_{2} !}{n ! l !}} 2^{-[(n+l) / 2]} \\
& \times \sum_{k=0} n(-1)^{l-\ell_{1}+k}\left(\begin{array}{l}
n \\
k
\end{array}\right)\left(\begin{array}{c}
l \\
\ell_{1}-k
\end{array}\right) .
\end{aligned}
$$

For any isotropic interaction $\mathcal{V}$, we have

$$
\left\langle n, l|\mathcal{V}| n^{\prime} l^{\prime}\right\rangle=\delta_{n, n^{\prime}} \delta_{l, l^{\prime}} V_{l},
$$

and the potential is expressed by

$$
\begin{aligned}
\mathcal{V}= & \sum_{\ell_{1}, \ldots, \ell_{4}} \delta_{\ell_{1}+\ell_{2}, \ell_{3}+\ell_{4}} a_{\ell_{1}}^{\dagger} a_{\ell_{2}}^{\dagger} a_{\ell_{3}} a_{\ell_{4}} \\
& \times \sum_{n, l} \delta_{\ell_{1}+\ell_{2}, n+l} C_{\ell_{1}, \ell_{2}}^{n, l} C_{\ell_{3}, \ell_{4}}^{n, l} V_{l} .
\end{aligned}
$$

This decomposition of the interaction into Haldane pseudopotentials $V_{l}$ is similar to the partial wave approach employed in scattering theory. Each pseudopotential $V_{l}$ describes the interaction of a pair of particles with relative angular momentum $l$. In the sum over $l$, for symmetry reasons, only even (odd) terms contribute if the particles are spinless bosons (fermions). In the case where the atoms have additional internal degrees of freedom, denoted by an index $s$, which are conserved by the interaction, the potential generalizes to

$$
\begin{aligned}
\mathcal{V}= & \sum_{\ell_{1}, \ldots, \ell_{4}} \sum_{s, \tilde{s}} \delta_{\ell_{1}+\ell_{2}, \ell_{3}+\ell_{4}} a_{\ell_{1} s}^{\dagger} a_{\ell_{2} \tilde{s}}^{\dagger} a_{\ell_{3} s} a_{\ell_{4} \tilde{s}} \\
& \times \sum_{n, l} \delta_{\ell_{1}+\ell_{2}, n+l} C_{\ell_{1}, \ell_{2}}^{n, l} C_{\ell_{3}, \ell_{4}}^{n, l} V_{l}^{s \tilde{s}} .
\end{aligned}
$$

In the presence of such repulsive two-body potentials, Eqs. (7) and (8), a relative angular momentum in the motion of two particles is favored in order to reduce interaction energy. The price to pay for this increase of angular momentum is an energy increase due to the trapping potential. Upon tuning either the trapping frequency or the interaction strength, the system therefore undergoes several phase transitions, from the condensate wave function, Eq. (3), to strongly correlated phases. The most relevant trial wave functions to describe these phases will be presented in the next section.

\section{TOPOLOGICALLY ORDERED PHASES AND THEIR EXCITATIONS}

\section{A. Laughlin states}

The most famous trial state in the context of fractional quantum Hall systems is the Laughlin state. The wave function is given by

$$
\Psi_{\mathrm{L}}^{q}\left(z_{1}, \ldots, z_{N}\right) \propto \prod_{i<j}\left(z_{i}-z_{j}\right)^{q} \exp \left(-\sum_{i}\left|z_{i}\right|^{2} / 2\right) .
$$

The integer parameter $q$ fixes the total angular momentum of the state to $L_{q}=\frac{q}{2} N(N-1)$. This angular momentum is distributed such that the relative motion of each pair of particles carries $q$ units of angular momentum. Thus, every particle is seen by the other particles as a vortex with vorticity two. In the thermodynamic limit, where a filling factor $v$, defined as the ratio between the number of particles and the number of vortices, characterizes the state. The Laughlin states are found at $v=1 / q$. Furthermore, the Laughlin states are zero-energy eigenstates of a potential as given in Eq. (7), if we set $V_{l}=0$ for $l \geqslant q$. This is due to the fact that there are no pairs of particles with relative angular momentum smaller than $q$ which could give a nonzero contribution to the interaction energy. If $V_{l}>0$ for $l<q$, no other state at smaller or the same total angular momentum has this property, and the Laughlin state becomes the true ground state for a sufficiently weak trapping potential. Thus, a pure $s$-wave scattering represents a parent Hamiltonian for the $v=1 / 2$ Laughlin state, and bosonic atoms in artificial gauge potentials therefore represent ideal systems for realizing this state. In Sec. V, we will also study a Hamiltonian with $s$ and $d$-wave interactions, which is a parent Hamiltonian for the Laughlin state at filling $v=1 / 4$.

\section{B. Halperin states}

In the bosonic Laughlin wave function, all anticorrelation terms $z_{i}-z_{j}$ have to be squared for symmetry reasons. In a system with contact interaction, however, this is not favorable from the energetic point of view, as squaring the anticorrelation terms solely increases angular momentum. For particles which are distinguishable through an internal degree of freedom, one thus is able to find zero-energy eigenstates of the interaction at $L<N(N-1)$. For systems with two internal states, these are the so-called (lmn)-Halperin states:

$$
\begin{aligned}
& \Psi\left(z_{1 \uparrow}, \ldots, z_{N_{\uparrow} \uparrow}, z_{1 \downarrow}, \ldots, z_{N_{\downarrow} \downarrow}\right) \\
& \sim \prod_{1 \leqslant i<j \leqslant N_{\uparrow}}\left(z_{i \uparrow}-z_{j \uparrow}\right)^{l} \\
& \times \prod_{1 \leqslant i<j \leqslant N_{\downarrow}}\left(z_{i \downarrow}-z_{j \downarrow}\right)^{m} \prod_{\substack{1 \leqslant i \leqslant N_{\uparrow} \\
1 \leqslant j \leqslant N_{\downarrow}}}\left(z_{i \uparrow}-z_{j \downarrow}\right)^{n} .
\end{aligned}
$$

Here and in the following, we omit the overall Gaussian common to any state. For bosons, the smallest possible angular momentum is obtained at $l=m=2$ and $n=1$. The condition $l=m$ implies that the state is a spin singlet, and its filling factor is given by $v=2 / 3$.

\section{Composite Fermion states}

A powerful picture to explain the fractional quantum Hall physics is given in terms of composite fermions [53]. This theory interprets the term $\Phi=\prod_{i \neq j}\left(z_{i}-z_{j}\right)$ as an attachment of a vortex to each particle. These composite particles, by their exchange statistics fermions, may then form a noninteracting 
integer quantum Hall state, obtained simply by a Slater determinant $\Phi_{L^{\prime}}$ over the $N$ lowest single-particle levels which sum up to a total angular momentum $L^{\prime}$. The total angular momentum of the state is then given by $L=L^{\prime}+\frac{1}{2} N(N-1)$. For instance, if $L^{\prime}=\frac{1}{2} N(N-1)$, we obtain the same total angular momentum as for the 1/2-Laughlin state, and in fact, the energetically best choice is to put all composite particles into the LLL (running from $\ell=0$ to $\ell=N-1$ ), so one obtains $\Phi_{L^{\prime}}=\Phi$, and the full wave function $\Psi=$ $\Phi \Phi_{L^{\prime}=N(N-1) / 2}$ becomes identical to the Laughlin state.

Apart from the Laughlin state, the composite fermion theory produces a series of states at smaller angular momentum, and most of them turn out relevant for describing bosonic gases in artificial gauge fields $[40,56]$. One simply has to choose $L^{\prime}<\frac{1}{2} N(N-1)$, which becomes possible if higher Landau levels are involved, also allowing for single-particle states with negative angular momentum, $\ell \geqslant-n_{\mathrm{LL}}$, where $n_{\mathrm{LL}}$ numbers the Landau level (starting with 0 for the LLL). For that reason, the composite fermion wave function in general has to be reprojected into the LLL:

$$
\Psi_{\mathrm{CF}}^{L}\left(z_{1}, \ldots, z_{N}\right)=\mathcal{P}_{\mathrm{LLL}} \Phi_{L^{\prime}}\left(z_{1}, \ldots, z_{N}\right) \Phi\left(z_{1}, \ldots, z_{N}\right) .
$$

For some $L$, there might be different ways to occupy the single-particle levels at the same energy. In these cases, one can construct competing composite fermion states. In the thermodynamic limit, the composite fermion wave functions describe states at filling factor $v=n /(n+1)$, with $n$ the number of occupied LLs. Below, we will take a closer look into the composite fermion wave function at $L=N(N-2)$, representing the incompressible phase which is next to the Laughlin phase at $L=N(N-1)$. The composite fermion wave function for $L=N(N-2)$ is obtained by putting all but one composite fermion in the LLL, and one composite fermion into the $\ell=-1$ state of the first excited LL.

\section{Excitations}

The topological nature of a quantum state can be related to certain striking properties of its excitations. Quite generally, one distinguishes between low-lying (or gapless) edge excitations and gapped quasiparticle excitations. The edge excitations are further characterized by the finite amount of angular momentum (of the order of one) which is added to the ground state, whereas quasihole (quasiparticle) excitations change angular momentum by an amount of order $N$.

It has been argued that the form of the edge states characterizes the topological nature of a state [57]. For Laughlin-like phases, one has [58]

$$
\Psi_{\text {edge }}=P\left(z_{1}, \ldots, z_{N}\right) \Psi_{\mathrm{L}}^{q}\left(z_{1}, \ldots, z_{N}\right),
$$

where $P$ is any symmetric polynomial on the variables $\left\{z_{i}\right\}$. It is easy to check that if $\Psi_{\mathrm{L}}^{q}\left(z_{1}, \ldots, z_{N}\right)$ is a zero-energy eigenstate of the potential $\mathcal{V}$, also $P\left(z_{1}, \ldots, z_{N}\right) \Psi_{\mathrm{L}}^{q}\left(z_{1}, \ldots, z_{N}\right)$ will be one. To count the number of edge excitations at a given angular momentum difference $\delta L=L-L_{q}$, we should note that the symmetric polynomials are generated by $s_{n} \equiv \sum_{i} z_{i}^{n}$. Thus, for $\delta L=1$, there is a single edge state given by $s_{1} \Psi_{\mathrm{L}}$, for $\delta L=2$, there are two edge states given by $s_{2} \Psi_{\mathrm{L}}$ and $s_{1}^{2} \Psi_{\mathrm{L}}$, and so on. This sequence can be taken as a fingerprint of the topological phase of a system [57] and implies a degeneracy of the edge states given by $\mathcal{P}(\delta L)$, which is the number of partitions of $\delta L$ (the number of ways in which up to $N$ non-negative integers add up to $\delta L$ ) [58]. It is worth noting that long-range interactions generally lift the degeneracy of different edge states, with the possibility of mixing edge states with other gapped excitations.

In contrast to the edge excitations, there exist also localized excitations in form of local density increases (quasiparticles) or local density decreases (quasiholes) at a position $\xi$. The corresponding wave functions read

$$
\begin{aligned}
& \Psi_{\mathrm{qp}} \sim \prod_{i}\left(\partial_{i}-\xi\right) \Psi \equiv O_{\mathrm{qp}}\left(\partial_{i}, \xi\right) \Psi, \\
& \Psi_{\mathrm{qh}} \sim \prod_{i}\left(z_{i}-\xi\right) \Psi \equiv O_{\mathrm{qh}}\left(z_{i}, \xi\right) \Psi .
\end{aligned}
$$

Here, we introduce "particle" and "hole" operators, $O_{\mathrm{qp}}$ and $O_{\mathrm{qh}}$, which acting on any state create a quasiparticle or a vortex. We note that both $\Psi_{\mathrm{qh}}$ and $\Psi_{\mathrm{qp}}$ are states with explicitly broken rotational symmetry, unless $\xi=0$.

Moving $\xi$ adiabatically on a closed loop enclosing the area $A$, the wave function acquires a Berry phase. If the system is sufficiently homogeneous, we can write this phase factor as $\exp \left[i q_{\mathrm{eff}} A\right]$, defining an effective charge $q_{\mathrm{eff}}$ of the quasiparticle. We will calculate this effective charge in the following section for small-sized systems. Small dependence of $q_{\text {eff }}$ on the enclosed area suggests that the system has a topological bulk. For topologically ordered states, a striking feature is given by the fact that the effective charge is a fractional multiple of the elementary charge. In the case of the Laughlin state, for instance, one has $q_{\mathrm{eff}}=e / q$ [3].

Repeatedly applying the operators $O_{\mathrm{qh}-\mathrm{qp}}$ at sufficiently distinct $\xi$, one obtains states with several quasihole-quasiparticle excitations. It then becomes possible to test the statistical behavior of these excitations by wrapping one around the other, or simply adiabatically changing the positions $\xi_{1}$ and $\xi_{2}$ of two excitations, such that they interchange their position. Again, this procedure will add a phase factor to the wave function, which can be written as $\exp \left[i q_{\text {eff }} A+i \varphi_{\text {stat }}\right]$. Additionally to the charge term, we now also have a second contribution in the exponent stemming from the statistical phase angle $\varphi_{\text {stat }}$. This phase angle $(\bmod 2 \pi)$ is 0 for bosons, and $\pi$ for fermions. Quasihole or quasiparticle excitations of topologically ordered states are known to have a fractional statistical phase. Such particles are therefore classified as anyons [22]. For the Laughlin states, we have $\varphi_{\text {stat }}=\pi / q$.

\section{EFFECTIVE CHARGE AND STATISTICAL PHASE}

\section{A. Method}

An exact analytic calculation of the effective charge and statistical angle of the excitations for small Laughlin states $(N \leqslant 6)$ was presented in Ref. [48], in contrast with the standard literature (cf. [3,5,22]) where the effective charge and the statistical angle are computed in the thermodynamic limit. For small systems, one has to determine the function $f(\xi)=\left\langle\Psi_{\mathrm{Lqh}}(\xi)\left|\nabla_{\xi}\right| \Psi_{\mathrm{Lqh}}(\xi)\right\rangle$, where $\Psi_{\mathrm{Lqh}}$ denotes the 
quasihole excited Laughlin state. This in principle requires integrating all coordinates $z_{i}$. In Ref. [48], we avoided this multidimensional integration by decomposing the state into a many-body Fock basis [59]. Finally, one obtains the Berry phase $\gamma$ by simply evaluating a line integral $\gamma=\oint_{A} f(\xi) d \xi$.

In this paper, we choose a slightly different approach, as we are interested in the behavior of quasihole excitations above generic states obtained by exact diagonalization. That is, we consider any state given in the Fock basis

$$
|\Psi\rangle=\sum c_{i}|i\rangle,
$$

where $|i\rangle=\left|\ell_{1}, \ldots, \ell_{N}\right\rangle$ denotes the Fock states of $N$ particles with angular momenta $\ell_{1}, \ldots, \ell_{N}$. To generate the quasihole state, we have to apply $O_{\mathrm{qh}}(\xi)$ to each Fock state. We find

$$
\begin{aligned}
& O_{\mathrm{qh}}(\xi)\left|\ell_{1}, \ldots, \ell_{N}\right\rangle \\
& =\sum_{m=0}^{N}(-\xi)^{N-m} 2^{m / 2} \sum_{\left\{p_{i}\right\}} \sqrt{\frac{\left(\ell_{1}+p_{1}\right) ! \cdots\left(\ell_{N}+p_{N}\right) !}{\ell_{1} ! \cdots \ell_{N} !}} \\
& \quad \times\left|\ell_{1}+p_{1}, \ldots, \ell_{N}+p_{N}\right\rangle .
\end{aligned}
$$

Here, the sum over $p_{i}$ contains all choices of $p_{i}=0,1$ with $\sum_{i} p_{i}=m$.

With this, it is straightforward to calculate $\left|\Psi_{\mathrm{qh}}\right\rangle$ for any $|\Psi\rangle$ given in the Fock basis. For simplicity, we will assume that the quasiholes are moved at a fixed radial position in a rotational symmetric system. The Berry phase $\oint\left\langle\Psi_{\mathrm{qh}}(\xi)\left|\nabla_{\xi}\right| \Psi_{\mathrm{qh}}(\xi)\right\rangle d \xi$ is then given by

$$
\gamma=2 \pi \lim _{\Delta \phi \rightarrow 0} \frac{\left\langle\Psi_{\mathrm{qh}}(\phi) \mid \Psi_{\mathrm{qh}}(\phi+\Delta \phi)\right\rangle-1}{\Delta \phi},
$$

where $\phi$ denotes the angular position of the quasihole. It is worth noting that for a large class of many-body states which are rotationally symmetric $\gamma$ solely depends on $R$ and not on $\phi$. Dividing $\gamma$ by $R^{2}$, we obtain $q_{\text {eff }}$. To obtain $\varphi_{\text {stat }}$, we apply $O_{\mathrm{qh}}$ twice at opposite positions $\phi$ and $\phi+\pi$, and then move both quasiholes simultaneously to $\phi+\Delta \phi$ and $\phi+\pi+\Delta \phi$, until they have interchanged their positions.

\section{B. Results for the contact potential}

We will now exactly calculate the anyonic properties of vortices in the fractional quantum Hall wave functions introduced above. By comparison of these results for small systems with the known results in the thermodynamic limit, we find that despite significant finite-size effects, anyonic properties and thus topological order are established already in systems as small as $N \approx 6$.

\section{Laughlin states}

The exact ground state of a single component bosonic system with contact $s$-wave atom-atom interactions at $L=$ $N(N-1)$ is the Laughlin state, Eq. (9), for $q=2$. As described above, we introduced up to two vortices into the ground state wave function, and calculated the effective charge and braiding angle for different system sizes, $4 \leqslant N \leqslant 6$. The results are shown in Figs. 1(a) and 1(c): A single vortex at any radial position $R$ sufficiently far from the edge has an effective charge which is close to $1 / 2$, the value expected for the thermodynamically large system. Naturally, the effective
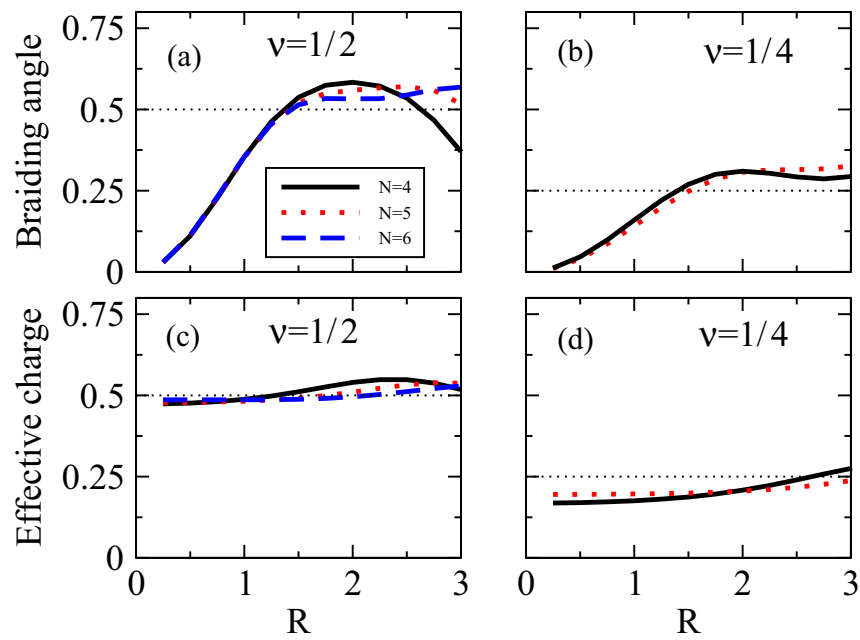

FIG. 1. (Color online) Statistical phase angle and effective charge of vortices in the Laughlin states at $L=N(N-1)$ [(a),(c)] and $L=2 N(N-1)[(\mathrm{b}),(\mathrm{d})] . R$ is the distance between the hole(s) and the center of the cloud (in units $\lambda$ ).

charge drops when the particle density diminishes at the edge of the system. The same is true for the braiding angle of two quasiholes, but here one also has to assure that they are sufficiently far from each other. Two vortices close to the center overlap with each other, distorting the value for the statistical phase. This makes it difficult to extract the bulk behavior for very small systems. The braiding angle for $N=4$ is basically a monotonously growing function of $R$, until a value close to $1 / 2$ is reached and the function starts to monotonously decrease again. However, by comparing the curves for different numbers of particles, one clearly finds that a plateau is formed near $1 / 2$, that is, a sizable bulk with the expected anyonic property is formed.

As shown in Figs. 1(b) and 1(d), the properties of vortices in small-sized $q=4$ Laughlin states differ more strongly from the thermodynamically expected values of $1 / 4$. Nevertheless, a comparison of the figures for four and five particles shows that the numbers quickly evolve in the expected direction. Due to the large angular momentum of this state, the Hilbert space grows too fast with $N$ to consider larger systems. On the other hand, even for $N=5$, the nonmonotonic behavior of the statistical phase suggests that an extended bulk has developed.

\section{Composite fermion state vs Laughlin quasiparticle state at $L=N(N-2)$}

If one increases the trap frequency (or decreases the interaction strength), at some critical value the system undergoes a transition from the Laughlin state to a state where the total angular momentum is decreased by $N$ units to $L=N(N-2)$ $[40,43,48]$. At this angular momentum, one can construct a composite fermion state, Eq. (11), by putting all but one composite particle into the lowest Landau level. Accordingly, this composite fermion state describes a system at filling factor $v=2 / 3$, and the vortex excitation (despite not being the elementary excitations of this state) should carry this effective charge and fractional statistics [60]. It has been shown that the exact ground state has very large overlap with 
this composite fermion state $(\gtrsim 0.99)$ for a small number of particles [40]. On the other hand, at $L=N(N-2)$ one can also construct a quasiparticle excitation of the Laughlin state according to Eq. (13), with the quasiparticle at the center, $\xi=0$. Considering system sizes of $4 \leqslant N \leqslant 7$ particles, we also find good overlaps $(\gtrsim 0.97)$ between this quasiparticle state and the true ground state. A vortex in the Laughlin quasiparticle state, however, should be of the Laughlin type, that is, showing 1/2-type anyonic properties.

Thus we encounter a situation where simply from overlap arguments it is not yet clear which topological properties can be expected, as two trial states describing different topological phases compete with each other. It is obvious that both composite fermion and Laughlin quasiparticle state also have very large overlaps one with another. One could expect that in such a situation the topological properties are not well defined unless we go to larger systems. However, at least with respect to the fractional charge of a vortex in the true ground state, shown in Figs. 2(a) and 2(c), the result of our direct diagonalization of the Hamiltonian approaches $2 / 3$ when increasing the number of particles from 4 to 7 . On the other hand, the braiding angle does not yet show a clear bulk behavior for $N=6$.

It is interesting to compare these results with the ones shown in Figs. 2(b) and 2(d), obtained for the Laughlin quasiparticle wave function. Despite the sizable overlap with the true ground state, the vortex charge is shifted towards a significantly lower value around 0.57 for $N=7$, and thus closer to the expected value $1 / 2$ than to the composite fermion value $2 / 3$. As the Laughlin quasiparticle state has, compared to the Laughlin state, a locally increased density around the center, it is not
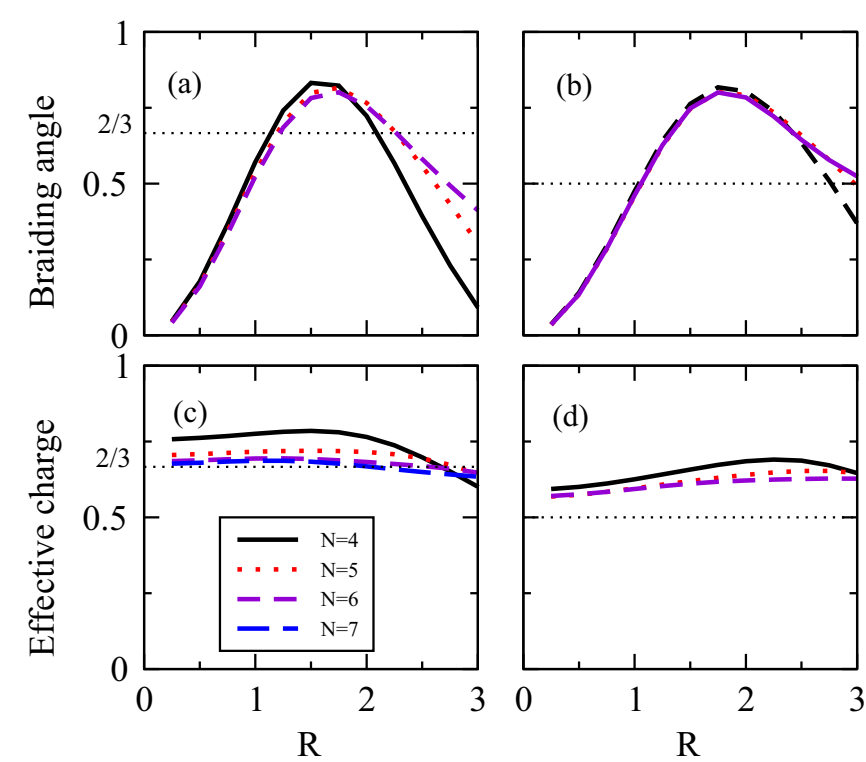

FIG. 2. (Color online) Statistical phase angle and effective charge of vortices in the $L=N(N-2)$ sector. The ones on the exact ground state are depicted in panels (a) and (c), while the corresponding ones for the Laughlin quasiparticle state with $\xi=0$ are depicted in (b) and (d). $R$ is the distance between the hole(s) and the center of the cloud (in units $\lambda$ ).
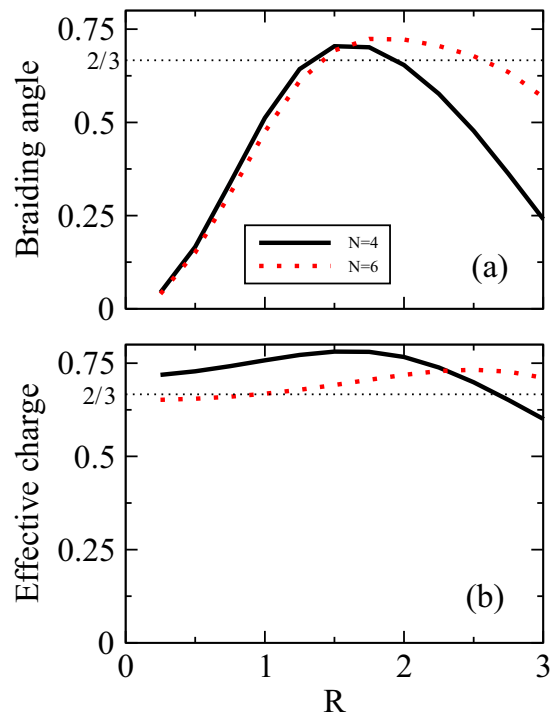

FIG. 3. (Color online) Statistical phase angle (a) and effective charge of vortices (b) in the (221)-Halperin state. $R$ is the distance between the hole(s) and the center of the cloud (in units $\lambda$ ).

surprising that also the effective charge of a nearby vortex is increased.

\section{3. (221)-Halperin state}

To conclude this section, let us finally present the charge and statistics of vortices in the (221)-Halperin state, Eq. (10). For this state, which is the spin-singlet generalization of the 1/2-Laughlin state, the elementary excitations are given in terms of vortices (and quasiparticles). As the (221)-Halperin state describes a system at $v=2 / 3$, we expect the fractional properties of the vortices to be given by that number. In fact, as shown in Fig. 3, for $N=6$, the effective charge has converged towards this value, and the braiding angle function is about to exhibit a plateau slightly above $2 / 3$.

\section{PERTURBING THE BOSONIC 1/2-LAUGHLIN STATE}

In the previous section, we have analyzed different fractional quantum Hall states which are obtained as ground states of a two-body contact interaction in a Hilbert space spanned by the lowest Landau level at a given $L$. For $L=N(N-1)$, one obtains the 1/2-Laughlin state as the unique zero-energy eigenstate with clear signatures of anyonic order even for small $N$. In this section, we analyze the robustness of these properties against deformations.

To this aim, we will study a Hamiltonian which differs from the parent Hamiltonian $\mathcal{H}_{\text {parent }}=\sum_{i} H_{i}+\mathcal{V}_{0}$ by some controllable perturbation. With this, the ground state of the Hamiltonian will, quite generally, read $\Psi_{\mathrm{GS}}=\alpha \Psi_{\mathrm{L}}+\beta \Psi_{\text {corr }}$, with $\alpha$ and $\beta$ nonzero parameters depending on the strength of the perturbation.

In Ref. [43], a similar study has been performed, motivated by the fact that a laser-induced gauge field for atoms is equivalent to a real magnetic field only after an adiabatic approximation. The nonadiabatic effects can then be taken as a perturbation to the parent Hamiltonian, breaking the 
rotational symmetry. The ground state is then a superposition of polynomials at different $L$, and in Ref. [43] it has been approximated by a wave function $\Psi_{\mathrm{L}}\left(\alpha+\beta \sum z_{i}^{2}\right)$. Thus, in that case, the correction to the Laughlin state is given by an edge excitation of the Laughlin state [cf. Eq. (12)]. From this it is directly clear that the bulk physics remains unchanged, and quasiholes which are sufficiently far from the edge maintain the Laughlin-like behavior. In this sense, the system considered in Ref. [43] trivially realizes an extended Laughlin phase.

The situation which we wish to study now is less trivial, as we will assume a perturbation which does not break any symmetry. Thus, the ground state $\Psi_{\mathrm{GS}}$ is obtained within the same Hilbert space as the Laughlin state $\Psi_{\mathrm{L}}$. Can the anyonic properties be maintained in such a scenario? We will study this question by adding a finite-range interaction, in terms of a nonzero Haldane pseudopotential $\mathcal{V}_{2}$ to the Hamiltonian. Such a model interaction approximates well a combination of a contact potential and a rapidly decaying long-range potential such as $r^{-3}$ dipolar interactions. By changing the strength of one potential relatively to the other, the ratio $V_{2} / V_{0}$ of the two pseudopotential strengths can be tuned. In particular, by making the $s$-wave scattering attractive, it is also possible to make $V_{2} \geqslant V_{0} \geqslant 0$. A study on the torus [61] has shown that a symmetry-breaking phase transition at $V_{2} / V_{0} \approx 0.5$ brings such system from a Laughlin-like phase to different vortex lattice phases. In this paper, we exclude the option of symmetry breaking by fixing the total angular momentum to $N(N-1)$, that is, regardless of the possibility to lower energy by increasing angular momentum, we study how a Laughlin system evolves when finite-range interactions are turned on.

\section{A. Braiding of excitations}

In order to get a quick overview of the quasihole properties when finite-range interactions are switched on, we plot for $N=6$ in Fig. 4 average values of the effective charge and

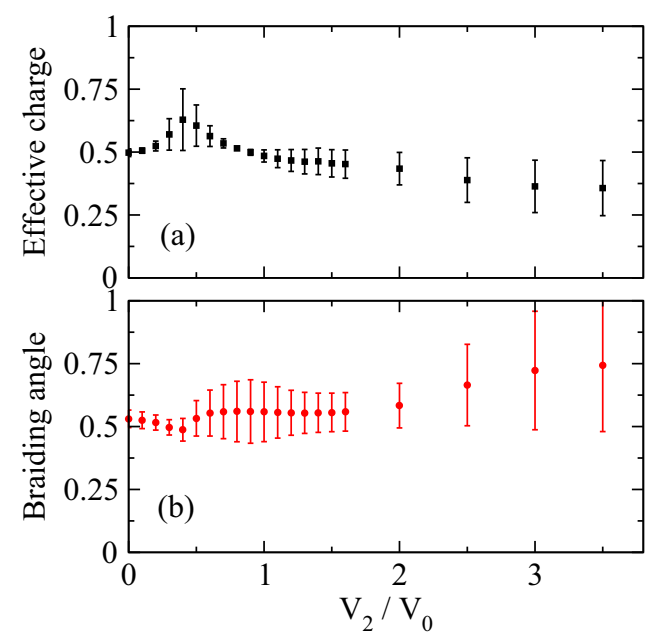

FIG. 4. (Color online) Effective charge (a) and statistical phase angle (b) of vortices averaged over different vortex positions. The represented value is the average \pm the standard deviation, in a $v=\frac{1}{2}$ Laughlin system $(N=6, L=30)$ perturbed by finite-range interactions characterized by a Haldane pseudopotential with relative strength $V_{2} / V_{0}$.

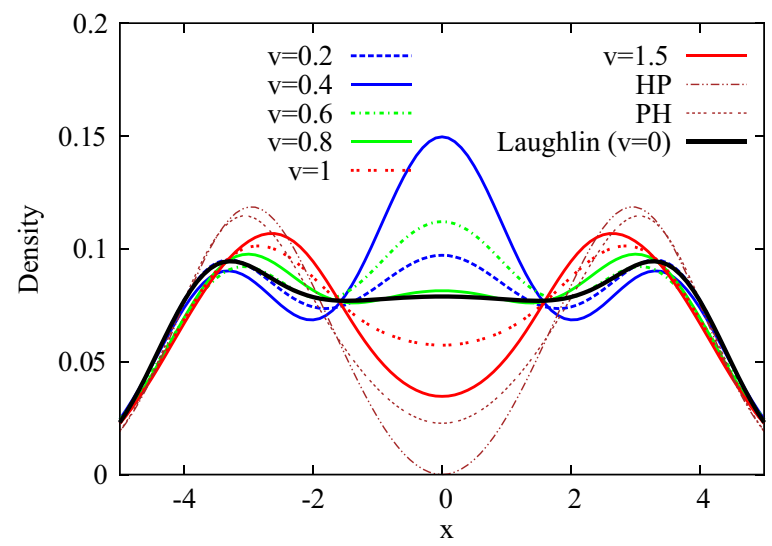

FIG. 5. (Color online) Density profiles (in units $\lambda^{-2}$ ) of the ground state of the system $(N=6, L=30)$ for different Haldane pseudopotential strengths $v=V_{2} / V_{0}$. The curves denoted by HP and $\mathrm{PH}$ show the density profiles of a trial wave function obtained from the Laughlin by adding a quasiparticle and subsequently a quasihole (PH) or a quasihole and subsequently a quasiparticle (HP) in the origin.

the braiding angle as a function of $V_{2} / V_{0}$, where the average is about the radial position of the quasiholes. We consider the interval $R \in[0.25,3]$ for the charge, and the interval $R \in[1.25,3]$ for the statistical phase, in steps of $\Delta R=0.25$. In particular, we display an error bar for each point in the figure given by its standard deviation in the interval. In this way we can discern between genuine fractional behavior, which has well-defined bulk properties and thus a small standard deviation, and other regimes. For instance, as we saw in Fig. 1, for the Laughlin state of $N=6$, the fractional charge and statistical phase are mostly constant in a broad region of $R$. This is reflected in a very small standard deviation of the charge and braiding angle at $V_{2} / V_{0}=0$ in Figs. 4(a) and 4(b).

For small values of $V_{2} / V_{0}<0.4$ the situation is very similar to the pure Laughlin state, with robust fractional properties of the ground state. At $V_{2} / V_{0} \approx 0.4$, the situation changes, and the average effective charge reaches its global maximum at $q_{\mathrm{eff}} \approx 0.64$, that is, almost $30 \%$ off the Laughlin value, and at the same time also its standard deviation increases dramatically. For larger values of $V_{2} / V_{0}$, the effective charge decreases monotonically, and also its standard deviation goes back to a very small value (around $V_{2} / V_{0} \approx 0.8$ ) before then increasing again. This behavior can be traced to density changes of the system upon increasing $V_{2} / V_{0}$, shown in Fig. 5 . While the Laughlin system, characterized by a homogeneous bulk density, first develops a density peak in the center, when $V_{2} / V_{0}$ is increased to 0.4 , this peak then continuously shrinks down to a density minimum for larger $V_{2} / V_{0}$. During this shrinking process, the system goes through a state of homogeneous density around $V_{2} / V_{0} \approx 0.8$.

The braiding properties turn out to be Laughlin-like up to a value $V_{2} / V_{0} \approx 0.4$, where the averaged braiding angle has a global minimum around $V_{2} / V_{0}=0.49$. The standard deviation still remains small, in contrast to the large standard deviation of the fractional charge. This indicates that the braiding angle would be a more robust property to characterize the topological properties of the system in this case. For larger 


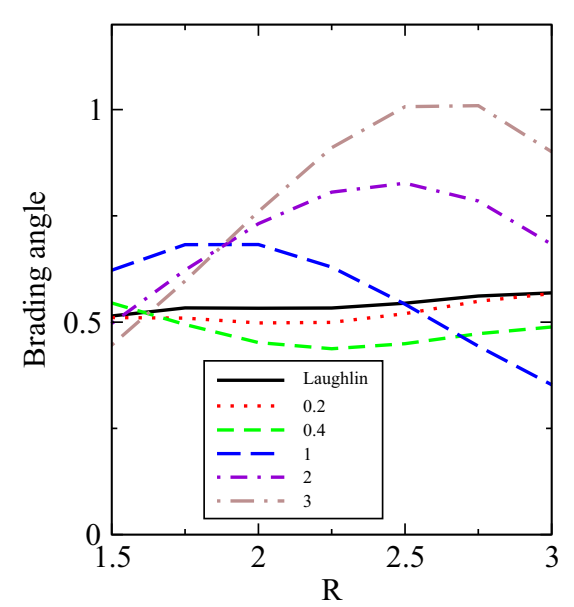

FIG. 6. (Color online) Statistical phase $(N=6, L=30)$ as a function of radial vortex position $R$ (in units $\lambda$ ) for different Haldane pseudopotential strengths. The labels correspond to the values of $V_{2} / V_{0}$.

values of $V_{2} / V_{0}$ the braiding angle increases slightly, but remains close to $1 / 2$ up to $V_{2} / V_{0} \approx 1.5$. One has to note, however, that for any $V_{2} / V_{0} \gtrsim 0.5$, the standard deviation of the braiding angle is significantly higher than in the Laughlin regime. To illustrate this point, we have explicitly plotted in Fig. 6 the braiding angle as a function of the vortex position for different values of $V_{2} / V_{0}$. For $V_{2} / V_{0}<0.4$ the curves are Laughlin-like, with nonmonotonic behavior around an average value close to 0.5 , indicating the formation of a bulk. The curves for $V_{2} / V_{0} \simeq 1$ despite having an average value close to $1 / 2$, do not show any bulk behavior. They monotonically increase to some maximum value significantly above $1 / 2$, and then monotonically decrease until reaching the edge of the system. This behavior results in an increased standard deviation. For large $V_{2} / V_{0}$ the curves further increase, leading to average values above the Laughlin mean, from 0.55 at $V_{2} / V_{0}=1.5$ to 0.75 at $V_{2} / V_{0}=3.5$.

Summarizing, Laughlin-like behavior with respect to both fractional charge and statistics is found only up to some value $V_{2} / V_{0}$ below 0.4 , whereas the bulk behavior with respect to quasihole braiding is lost only for slightly higher values of $V_{2} / V_{0}$. The analysis of the excitation thus yields $V_{2} / V_{0}=$ 0.4 as a rough estimate of the topological phase transition. It suggests that there is no topological order in the system when the Laughlin order has been destroyed, but the size of the considered system $(N=6)$ might be too small to display the order. To back our interpretation so far, and get further insight into the physical behavior of the system, we will in the following discuss the overlaps, correlation functions, and the energy spectrum of the system.

\section{B. Overlaps, correlations, and spectral properties}

\section{Low-lying spectral properties}

A first quantity worth examining is the energy spectrum. We distinguish between the spectrum at constant angular momentum $L=N(N-1)$, and the edge spectrum in subspaces at higher $L$. We will increase the ratio $V_{2} / V_{0}$ keeping $V_{0}$ constant and increasing $V_{2}$. It is thus natural that we

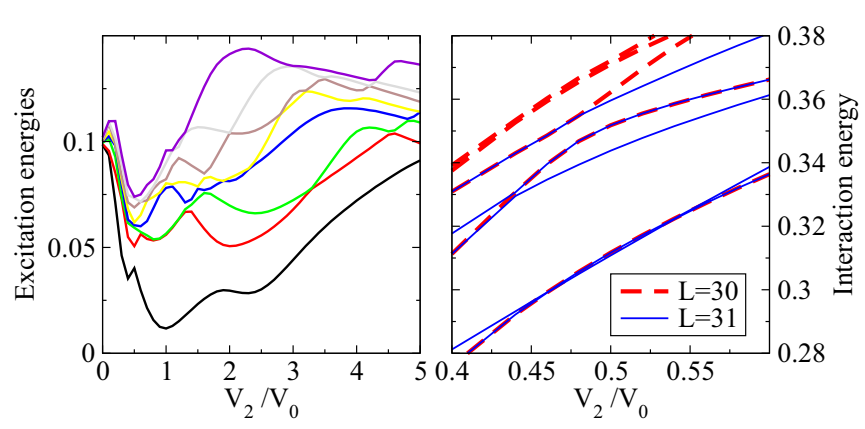

FIG. 7. (Color online) (a) Gaps (in units $V_{0}$ ) above ground state at $N=6$ and $L=30$ in the presence of a Haldane pseudopotential with relative strength $V_{2} / V_{0}$. (b) Interaction energy (in units $V_{0}$ ) of lowest states at $N=6$ and $L=30$ and $L=31$.

find that the energy of all levels increases with $V_{2} / V_{0}$. In contrast to this, the energy gap above the ground state in the $L=N(N-1)$ subspace turns out to be larger at $V_{2}=0$. Remarkably, as shown in Fig. 7(a), the gap never closes in the interval $0 \leqslant V_{2} / V_{0} \leqslant 5$. Thus, we do not obtain a clear hint for a phase transition in the form of a level crossing. The only signatures of some reorganization are the three minima of the gap. They can be traced back to avoided level crossings: The global minimum, at $V_{2} / V_{0} \approx 1$, coincides with an avoided level crossing between the ground state and its first excited state. Near the other minima, at $V_{2} / V_{0} \approx 0.4$ and $V_{2} / V_{0} \approx 2.4$, avoided level crossings between the second and the third level are pronounced, whereas the ground state level remains relatively far from the neighboring level.

While these observations do not suggest a phase transition, a different picture emerges from the edge spectrum, that is, the spectrum of $L$-changing excitations. Here, we restrict ourselves to $\Delta L=1$. For almost the full range $0 \leqslant V_{2} / V_{0} \leqslant$ 5 , we find the ground state energy at $L=N(N-1)$ to be the same as at $L=N(N-1)+1$, and the ground state at $L=N(N-1)+1$ is obtained from the ground state at $L=$ $N(N-1)$ as an edge state via the construction of Eq. (12). The gap above the ground state is slightly smaller in the $L=N(N-1)+1$ subspace, but of similar order in both subspaces. There is, however, one remarkable exception to this behavior, found in the interval $0.4 \leqslant V_{2} / V_{0} \leqslant 0.6$, depicted in Fig. 7(b). Here, the gap in the $L=N(N-1)+1$ subspace vanishes completely. Two nearby level crossings between the two lowest states even result in a small negative gap between the edge state and a second state. Since in quantum Hall effect the robust conductivity properties characterizing the different phases are attributed to the number of robust edge states, the occurrence of a second low-lying excitation strongly suggests a topological phase transition located in the interval $0.4 \leqslant V_{2} / V_{0} \leqslant 0.6$, in agreement with our considerations of the anyonic properties.

\section{Overlaps with perturbed Laughlin wave functions}

Next, we calculate the overlap of the exact ground state (for $N=6$ ) with the Laughlin state, shown in Fig. 8: It monotonously decreases with increasing $V_{2} / V_{0}$, but it remains above $>0.9$ up to values $V_{2} / V_{0} \approx 0.3$. For stronger $d$-wave interactions, the decay becomes significantly steeper. The 


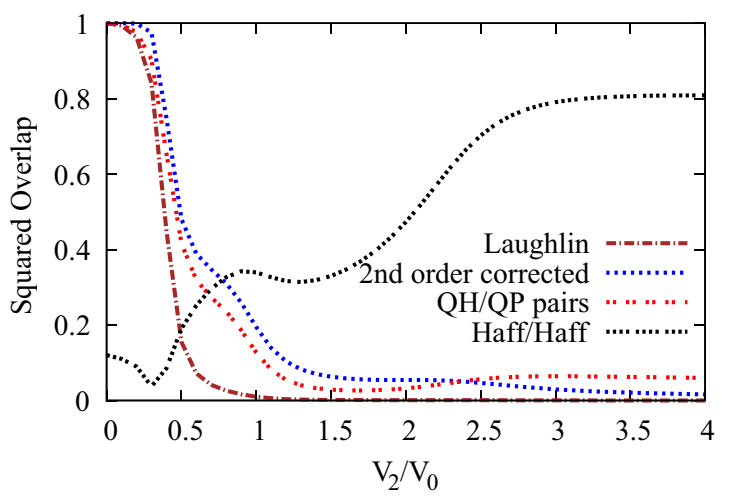

FIG. 8. (Color online) Overlaps of the exact ground state at $N=$ 6 and $L=30$ with different trial wave functions in the presence of a Haldane pseudopotential with relative strength $V_{2} / V_{0}$.

sharpest decline is found in the interval $0.4<V_{2} / V_{0}<0.5$, in which the overlap falls from 0.672 to 0.395 . This observation suggests a topological transition at $V_{2} / V_{0} \approx 0.4$, backing our estimate of the phase boundary obtained by analysis of the quasihole behavior.

The absence of a level crossing with respect to the ground state suggests the use of perturbation theory to describe the ground state. Making use of the numerical solutions at $V_{2} / V_{0}=0$, we may try to describe the ground state at finite $V_{2} / V_{0}$ as a perturbative state. As long as this ansatz yields good overlaps with the exact ground state, the system can be interpreted as a perturbed Laughlin system. As we also have exact knowledge of the ground state at finite $V_{2} / V_{0}$, we can directly check to which order in perturbation theory we get improvements. It turns out that for any $V_{2} / V_{1}>0.1$, results improve up to the second order, but then become worse. The reason is the quickly diverging nature of the perturbative expansion: Setting the smallness parameter $V_{2} / V_{0}=1$, the first-order corrections to the ground state, $\Psi^{(1)}$, are normalized to 0.48 . The second-order corrections, $\Psi^{(2)}$, are normalized to 1.26, whereas the third-order corrections are normalized to 139. We thus define

$$
\Psi_{\text {pert }} \sim \Psi_{\mathrm{L}}+\frac{V_{2}}{V_{0}} \Psi^{(1)}+\left(\frac{V_{2}}{V_{0}}\right)^{2} \Psi^{(2)},
$$

and plot the overlap of $\Psi_{\text {pert }}$ with the exact ground state in Fig. 8. Perturbation theory works very accurately up to $V_{2} / V_{0} \approx 0.3$ with overlaps $\gtrsim 0.99$. The results at larger $V_{2} / V_{0}$ become increasingly worse, and the steepest decay occurs between $0.4 \leqslant V_{2} / V_{0} \leqslant 0.5$, as has already been the case for the overlap of the exact ground state with the unperturbed Laughlin state. For $V_{2} / V_{0}=0.5$, the squared overlap has dropped below 0.5 , that is, the dominant contribution to the ground state can no longer be understood as a perturbed Laughlin state.

While the perturbative ansatz yields a good description of the ground state for sufficiently small $V_{2} / V_{0}$, it does not tell much about the nature of the deformations. To get a physical picture, we construct different trial states. One possibility of deforming a Laughlin state without changing its angular momentum is the creation of one quasiparticle-quasihole pair in the center, yielding the trial states $O_{\mathrm{qh}}(0) O_{\mathrm{qp}}(0) \Psi_{\mathrm{L}}$ and
$O_{\mathrm{qp}}(0) O_{\mathrm{qh}}(0) \Psi_{\mathrm{L}}$. Further states can be obtained by repeatedly generating quasiparticle-quasihole pairs at the center. In Fig. 8, we show the overlap of the ground state with a trial state constructed as the optimal superposition of the Laughlin state, the two states with one pair excitation, and the four states with two pair excitations. This construction provides a significant improvement compared to the pure Laughlin state, but is less accurate than $\Psi_{\text {pert }}$.

Why the system, in the presence of $d$-wave repulsion between the atoms, benefits from the creation of quasiparticlequasihole pairs can be understood by noting that this construction takes out anticorrelations from the state via the derivative associated with the quasiparticle construction, and reintroduces new anticorrelations via the $z$ 's of the quasihole construction. In this way, some pairs of particles gain relative angular momentum, and thus will avoid $d$-wave scattering, for the cost of an increased $s$-wave interaction between other pairs of particles. Alternatively, we may achieve a similar effect in a more explicit way by writing

$$
\Psi_{\text {trial }}=\sum_{\mathrm{P}} \frac{\left(z_{\mathrm{P}(1)}-z_{\mathrm{P}(2)}\right)^{2}}{\left(z_{\mathrm{P}(3)}-z_{\mathrm{P}(4)}\right)^{2}} \Psi_{\mathrm{L}},
$$

with $\mathrm{P}$ denoting permutations. Optimizing the wave function $\Psi=\alpha \Psi_{\mathrm{L}}+\beta \Psi_{\text {trial }}$ yields similar results as the quasiholequasiparticle constructions, that is, it captures well the physics in the Laughlin-like regime, $V_{2} / V_{0} \lesssim 0.5$, but fails at larger values of $V_{2} / V_{0}$.

For larger values of $V_{2} / V_{0}$, it seems to be natural that more and more second-order anticorrelations are broken to free angular momentum for the creation of fourth-order anticorrelations. A wave function which pairwise removes anticorrelations from the Laughlin state is the Haffnian wave function [62]:

$$
\Psi_{\mathrm{Hf}} \sim \sum_{\mathrm{P}} \frac{1}{\left(z_{\mathrm{P}(1)}-z_{\mathrm{P}(2)}\right)^{2} \cdots\left(z_{\mathrm{P}(N-1)}-z_{\mathrm{P}(N)}\right)^{2}} \Psi_{\mathrm{L}}
$$

Similarly to the Pfaffian construction by Moore and Read [23] with $p$-wave pairing, the Haffnian wave function describes the formation of $d$-wave pairing. The Haffnian frees $N$ quanta of angular momenta which now can be used to generate fourthorder anticorrelations. A natural way for reintroducing angular momentum is by another Haffnian, $\operatorname{Hf}\left(z_{i}-z_{j}\right)^{2}$. In this way we obtain

$$
\begin{aligned}
\Psi_{\mathrm{Hf} / \mathrm{Hf}} \sim & {\left[\sum_{\mathrm{P}}\left(z_{\mathrm{P}(1)}-z_{\mathrm{P}(2)}\right)^{2} \cdots\left(z_{\mathrm{P}(N-1)}-z_{\mathrm{P}(N)}\right)^{2}\right] } \\
& \times\left[\sum_{\mathrm{P}} \frac{1}{\left(z_{\mathrm{P}(1)}-z_{\mathrm{P}(2)}\right)^{2} \cdots\left(z_{\mathrm{P}(N-1)}-z_{\mathrm{P}(N)}\right)^{2}}\right] \Psi_{\mathrm{L}} .
\end{aligned}
$$

Alternatively, one might consider a quasihole construction, that is, the wave function $\Psi_{\mathrm{Hf}}^{\mathrm{qh}} \sim O_{\mathrm{qh}}(\xi=0) \Psi_{\mathrm{Hf}}$, which yields a similar expression as $\Psi_{\mathrm{Hf} / \mathrm{Hf}}$, with overlap $\left|\left\langle\Psi_{\mathrm{Hf} / \mathrm{Hf}} \mid \Psi_{\mathrm{Hf}}^{\mathrm{qh}}\right\rangle\right|=$ 0.97 for $N=6$. Both wave functions yield squared overlaps $>0.5$ with the exact ground state for $V_{2} / V_{0} \gtrsim 2$, as shown in Fig. 8 . 

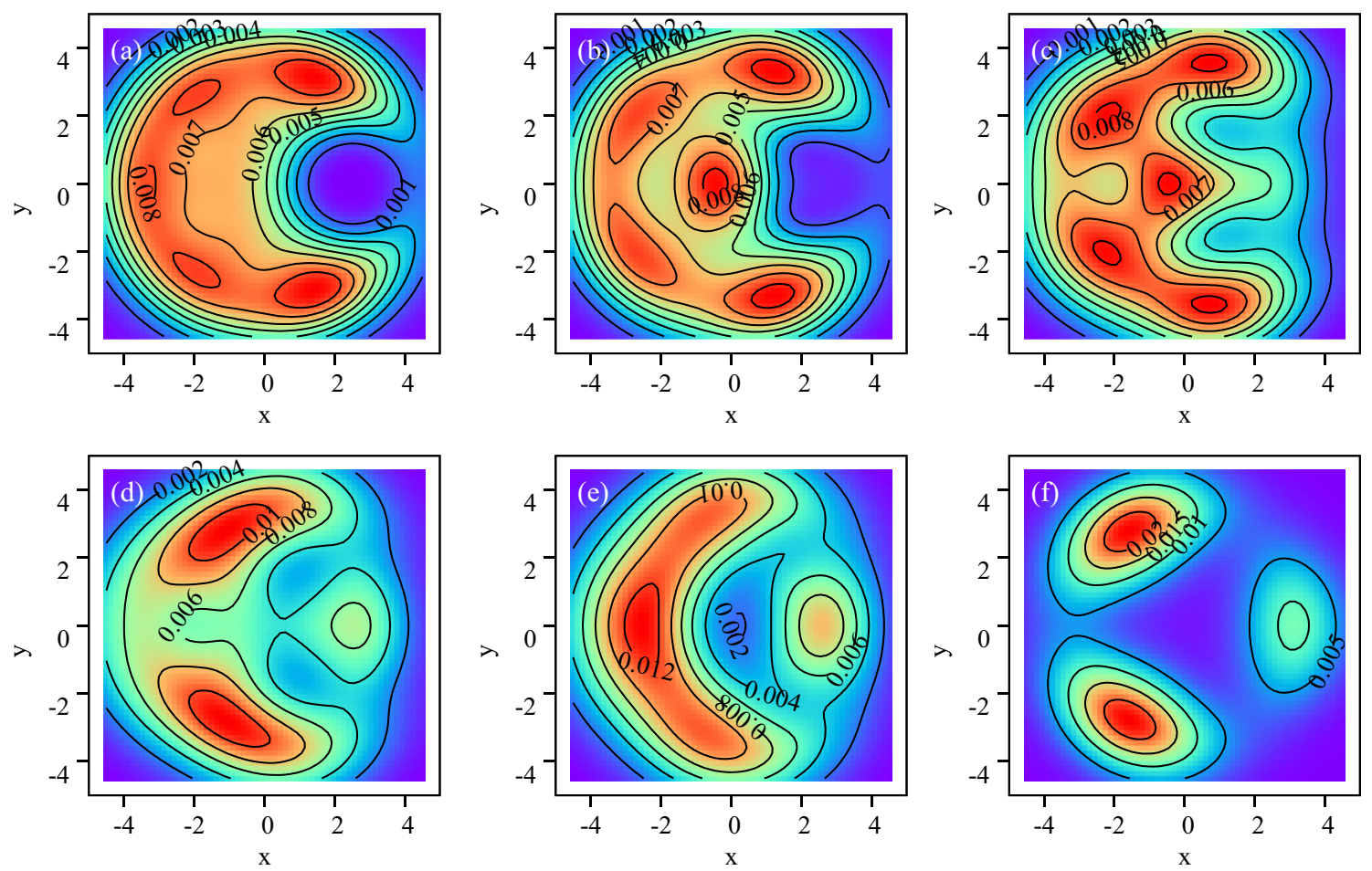

FIG. 9. (Color online) Ground state correlation functions for $N=6$ at different values of $V_{2} / V_{0}$ with one particle fixed to $(x, y)=(2.5,0)$ in units $\lambda$. Bright colors denote relatively large values of the correlation function, that is, a high probability of finding a particle at the respective position. From (a) to (f), $V_{2} / V_{0}$ takes the values $0,0.3,0.5,0.8,1.5$, and 4.0.

\section{Two-body correlation functions}

Many-body correlated states, e.g., the Laughlin, are known to exhibit their inner order in two-body correlation functions, despite the fact that no structure is seen in the one-body density. Here we present two-body correlation functions (see Fig. 9) for selected values of $V_{2} / V_{0}$ and $N=6$. The plots show the probability distribution of finding a particle at position $(x, y)$ when another particle has been fixed at $(x, y)=(2.5,0)$, a position of relatively high density for any value of $V_{2} / V_{0}$ (cf. Fig. 5).

In the Laughlin state, Fig. 9(a), the probability of finding two particles at the same position is zero. Apart from this strong anticorrelation, the state does not show pronounced correlation structures. Fixing one particle still allows the other particles to occupy the remaining area with some soft peaks near the edge of the system. The same peaks become more pronounced in Fig. 9(b) at $V_{2} / V_{0}=0.3$, showing that four particles tend to arrange on a semicircle at the edge, while the fifth is most likely to be found near the center. The anticorrelation of the Laughlin is still present. The similarity between the correlation patterns in (a) and (b) suggest that both states describe the same phase. In (c), at $V_{2} / V_{0}=0.5$, the peaks of (b) become more pronounced, but the anticorrelation structure is lost. Increasing $V_{2} / V_{0}$ even more, to 0.8 in (d), 1.5 in (e), or 4.0 in (f), it becomes more and more likely to find a second particle at the position $(2.5,0)$. In fact, the structure in $(d)$, with a peak at $(2.5,0)$ and two peaks on the opposite side of the cloud is a precursor of the pairing described by Eq. (21). As is seen from Fig. 8, the overlap with this wave function starts to grow for $V_{2} / V_{0}>0.5$. Interestingly, the correlation structure of (d) does not transform continuously into the correlation structure of (f), with three very pronounced peaks, and almost zero values between the peaks. Instead, at intermediate values, as shown in (e) for $V_{2} / V_{0}=1.5$, there is only one peak at $(2.5,0)$ and increased values forming a semicircle on the other side of the cloud. For $V_{2} / V_{0}=4$, the overlap with Eq. (21) is $\simeq 0.8$ (see Fig. 8). Accordingly we find good resemblance between the pair correlations in the ground state, Fig. 9(f), and the pair correlation of the trial state, shown in Fig. 10, which clearly features a similar pairing structure.

Summarizing this discussion, the anticorrelated nature of the Laughlin state is lost at $V_{2} / V_{0} \approx 0.5$, and the system

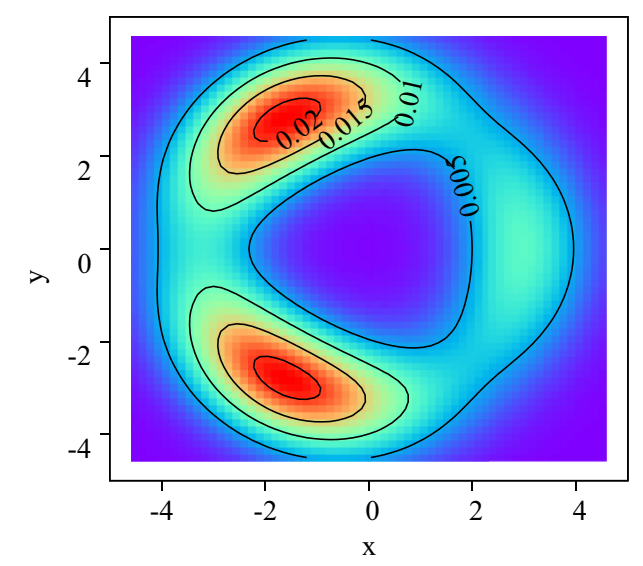

FIG. 10. (Color online) Pair correlation functions for $N=6$ for $\Psi_{\mathrm{Hf} / \mathrm{Hf}}$ given in Eq. (21) with one particle fixed to $(x, y)=(2.5,0)$ in units $\lambda$. 
undergoes an evolution to a state with strong pairing and strong anticorrelations between the pairs described well by the trial wave function, Eq. (21).

Let us also note that in the limit $V_{2} / V_{0} \rightarrow \infty$, the pairing state is not the only ground state of the system. For instance, a state $\Psi \sim Z^{N(N-1)}$ with $Z=\sum_{i} z_{i}$ is a zero-energy eigenstate of any potential with $V_{0}=0$, since all angular momentum is now simply attached to the center of mass. The number of other possibilities to achieve zero-energy states increases with $N$. This observation may seem to contradict the trend in the energy spectrum, Fig. 7. In this figure, the increase of the gap for large $V_{2} / V_{0}$ is due to the choice of a constant $V_{0}$ defining the units of energy. Since the limit $V_{2} / V_{0} \rightarrow \infty$ would then require $V_{2} \rightarrow \infty$, the gap becomes small in comparison to the dominant energy scale of the system.

\section{SUMMARY AND DISCUSSION}

In this paper we have studied few-body bosonic systems in the fractional quantum Hall regime, that is, in two dimensions, subjected to an external gauge field, and with repulsive atomatom interactions. We have presented exact diagonalization results for small systems, with the aim of understanding how the topological properties of the many-body states, well understood in the thermodynamic limit for certain trial wave functions presented in Sec. III, manifest in few-body systems.

Some of these "trial" states, notably the 1/2-Laughlin state for single component or the (221)-Halperin for two-component bosons, are actually exact ground states of the system with contact interactions. While in such a case it is a priori clear that the given state describes correctly the system of interest, it is not obvious how finite-size effects affect the topological features. Other trial states, for instance generic composite fermion states, have overlaps with the ground state of the system which decrease when the system size is increased. In this case, it is even harder to classify the topological order of the system by referring to some trial wave function. In this paper, by considering the fractionality of vortex excitations, that is, the fractional effective charge of a single vortex, and the fractional statistics of two vortices, we have directly employed one of the most intriguing properties of topological order, and have thereby characterized different quantum Hall phases.

Our analysis in Sec. IV has shown that topological features are displayed already in very small systems. Both effective charge and braiding angle tend towards the expected bulk value in the case of the 1/2-Laughlin state, the 1/4-Laughlin state, and the (221)-Halperin state for systems as small as $N=6$ particles. The most pronounced bulk is obtained for the $1 / 2$ Laughlin state. Furthermore, we were able to use our study of vortex properties to distinguish between a state describing a rotationally symmetric quasiparticle excitation of the $1 / 2$ Laughlin state from the composite fermion state at the same angular momentum $L=N(N-2)$. More precisely, we find that the effective charge of a vortex yields a good estimate of the filling factor, while no bulk is found with respect to the braiding of vortices. We note in this context that the quasihole charge, despite being related to the local density of the state, is not simply proportional to it. Thus, although the filling factor may already be estimated by a simple measurement of the density, such measurement is not equivalent to the measurement of the effective charge.

In Sec. V we considered a system described by the parent Hamiltonian of the Laughlin state plus a perturbation in form of $d$-wave scattering. Fixing the angular momentum to $L=$ $N(N-1)$, we have studied the evolution of the system when increasing the perturbation. We could show that the braiding of vortices yields a signal of the topological phase transition occurring in the system, seen as an increased sensibility of the braiding angle on the positions of the vortices. Our estimate of the phase boundary obtained by the braiding is in agreement with estimates based on different quantities such as the overlap with the Laughlin or Laughlin-like state, the number of edge excitations, and the energy spectrum at constant $L$. As the spectrum lacks ground state level crossings, we conclude that the topological phase transition occurs in a continuous way. In the regime where $d$-wave scattering is strong, we are able to describe the system by a $d$-wave paired state, which is constructed from the Laughlin state by applying two different forms of Haffnian terms. This trial state has increasingly large overlap and exhibits similar pairing features as observed in the two-body correlation function of the exact ground state.

Demonstrating how topological order reflects in anyonic properties of small-sized systems, our study provides information which can be useful for validating a quantum simulation of fractional quantum Hall systems. In particular, such experiments might allow for variable system sizes, ranging from the sizes considered here, accessible through classical computations, to large numbers outperforming any classical attempt. As the comparison of different sizes from 4 to 7 shows, one can expect rapid improvements of the results only by a slight increase of the system size. We thus conclude that the measurement of the effective charge and the braiding angle, e.g., by using the scheme provided in Ref. [46], is a powerful tool for classifying the topological order of a system. In particular, such experiments could for the first time observe fractional quantum statistics in a direct way.

\section{ACKNOWLEDGMENTS}

The authors thank N. Barberán for providing us with her manuscript on Haldane pseudopotentials. This work has been supported by EU (SIQS, EQUAM), ERC (QUAGATUA), Spanish MINCIN (Grant No. FIS2008-00784 TOQATA), Generalitat de Catalunya (Grant No. 2009-SGR1289), and Alexander von Humboldt Stiftung. B.J.D. is supported by the Ramón y Cajal program.
[1] K. von Klitzing, G. Dorda, and M. Pepper, Phys. Rev. Lett. 45, 494 (1980).
[2] D. C. Tsui, H. L. Stormer, and A. C. Gossard, Phys. Rev. Lett. 48, 1559 (1982). 
[3] R. B. Laughlin, Phys. Rev. Lett. 50, 1395 (1983).

[4] X. G. Wen and Q. Niu, Phys. Rev. B 41, 9377 (1990).

[5] X.-G. Wen, Quantum Field Theory of Many-Body Systems (Oxford University Press, New York, 2004).

[6] C. Nayak, S. H. Simon, A. Stern, M. Freedman, and S. Das Sarma, Rev. Mod. Phys. 80, 1083 (2008).

[7] E. Alba, X. Fernandez-Gonzalvo, J. Mur-Petit, J. K. Pachos, and J. J. Garcia-Ripoll, Phys. Rev. Lett. 107, 235301 (2011).

[8] N. Goldman, J. Dalibard, A. Dauphin, F. Gerbier, M. Lewenstein, P. Zoller, and I. B. Spielman, Proc. Natl. Acad. Sci. USA 110, 6736 (2013).

[9] A. Dauphin and N. Goldman, Phys. Rev. Lett. 111, 135302 (2013).

[10] A. P. Schnyder, S. Ryu, A. Furusaki, and A. W. W. Ludwig, Phys. Rev. B 78, 195125 (2008).

[11] A. Kitaev, Periodic Table for Topological Insulators and Superconductors, AIP Conf. Proc. No. (AIP, Melville, NY, 2009), p. 22.

[12] X. Chen, Z.-C. Gu, Z.-X. Liu, and X.-G. Wen, Phys. Rev. B 87, 155114 (2013).

[13] Y.-M. Lu and A. Vishwanath, Phys. Rev. B 86, 125119 (2012).

[14] M. A. Cazalilla, N. Barberán, and N. R. Cooper, Phys. Rev. B 71, 121303 (2005).

[15] H. Li and F. D. M. Haldane, Phys. Rev. Lett. 101, 010504 (2008).

[16] B. Estienne, Z. Papić, N. Regnault, and B. A. Bernevig, Phys. Rev. B 87, 161112 (2013).

[17] M. Levin and X.-G. Wen, Phys. Rev. Lett. 96, 110405 (2006).

[18] A. Kitaev and J. Preskill, Phys. Rev. Lett. 96, 110404 (2006).

[19] M. Haque, O. Zozulya, and K. Schoutens, Phys. Rev. Lett. 98, 060401 (2007).

[20] Y. Zhang, T. Grover, and A. Vishwanath, Phys. Rev. B 84, 075128 (2011).

[21] J. Zhao, D. N. Sheng, and F. D. M. Haldane, Phys. Rev. B 83, 195135 (2011).

[22] D. Arovas, J. R. Schrieffer, and F. Wilczek, Phys. Rev. Lett. 53, 722 (1984).

[23] G. Moore and N. Read, Nucl. Phys. B 360, 362 (1991).

[24] R. de Picciotto, M. Reznikov, M. Heiblum, V. Umansky, G. Bunin, and D. Mahalu, Nature (London) 389, 162 (1997).

[25] L. Saminadayar, D. C. Glattli, Y. Jin, and B. Etienne, Phys. Rev. Lett. 79, 2526 (1997).

[26] B. I. Halperin, A. Stern, I. Neder, and B. Rosenow, Phys. Rev. B 83, 155440 (2011).

[27] Y. Ji, Y. Chung, D. Sprinzak, M. Heiblum, D. Mahalu, and H. Shtrikman, Nature (London) 422, 415 (2003).

[28] H. Choi, P. Jiang, M. D. Godfrey, W. Kang, S. H. Simon, L. N. Pfeiffer, K. W. West, and K. W. Baldwin, New J. Phys. 13, 055007 (2011).

[29] V. Schweikhard, I. Coddington, P. Engels, V. P. Mogendorff, and E. A. Cornell, Phys. Rev. Lett. 92, 040404 (2004).

[30] N. Cooper, Adv. Phys. 57, 539 (2008).

[31] A. L. Fetter, Rev. Mod. Phys. 81, 647 (2009).

[32] D. Jaksch and P. Zoller, New J. Phys. 5, 56 (2003).
[33] J. Dalibard, F. Gerbier, G. Juzeliūnas, and P. Öhberg, Rev. Mod. Phys. 83, 1523 (2011).

[34] N. R. Cooper, Phys. Rev. Lett. 106, 175301 (2011).

[35] Y.-J. Lin, R. L. Compton, A. R. Perry, W. D. Phillips, J. V. Porto, and I. B. Spielman, Phys. Rev. Lett. 102, 130401 (2009).

[36] K. Jiménez-García, L. J. LeBlanc, R. A. Williams, M. C. Beeler, A. R. Perry, and I. B. Spielman, Phys. Rev. Lett. 108, 225303 (2012).

[37] M. Aidelsburger, M. Atala, S. Nascimbène, S. Trotzky, Y.-A. Chen, and I. Bloch, Phys. Rev. Lett. 107, 255301 (2011).

[38] N. Goldman, G. Juzeliūnas, P. Öhberg, and I. Spielman, arXiv:1308.6533.

[39] J. Struck, C. Ölschläger, M. Weinberg, P. Hauke, J. Simonet, A. Eckardt, M. Lewenstein, K. Sengstock, and P. Windpassinger, Phys. Rev. Lett. 108, 225304 (2012).

[40] N. R. Cooper and N. K. Wilkin, Phys. Rev. B 60, R16279 (1999).

[41] N. R. Cooper, N. K. Wilkin, and J. M. F. Gunn, Phys. Rev. Lett. 87, 120405 (2001).

[42] M. Hafezi, A. S. Sørensen, E. Demler, and M. D. Lukin, Phys. Rev. A 76, 023613 (2007).

[43] B. Juliá-Díaz, D. Dagnino, K. J. Günter, T. Graß, N. Barberán, M. Lewenstein, and J. Dalibard, Phys. Rev. A 84, 053605 (2011).

[44] T. Graß, B. Juliá-Díaz, N. Barberán, and M. Lewenstein, Phys. Rev. A 86, 021603 (2012).

[45] S. Furukawa and M. Ueda, Phys. Rev. A 86, 031604 (2012).

[46] B. Paredes, P. Fedichev, J. I. Cirac, and P. Zoller, Phys. Rev. Lett. 87, 010402 (2001).

[47] E. Kapit, P. Ginsparg, and E. Mueller, Phys. Rev. Lett. 108, 066802 (2012).

[48] B. Juliá-Díaz, T. Graß, N. Barberán, and M. Lewenstein, New J. Phys. 14, 055003 (2012).

[49] R. Umucallar and I. Carusotto, Phys. Lett. A 377, 2074 (2013).

[50] G. A. Fiete, G. Refael, and M. P. A. Fisher, Phys. Rev. Lett. 99, 166805 (2007).

[51] M. B. Tavernier, E. Anisimovas, and F. M. Peeters, Phys. Rev. B 74, 125305 (2006).

[52] H. Saarikoski, S. M. Reimann, A. Harju, and M. Manninen, Rev. Mod. Phys. 82, 2785 (2010).

[53] J. K. Jain, Phys. Rev. Lett. 63, 199 (1989).

[54] N. Read and E. Rezayi, Phys. Rev. B 59, 8084 (1999).

[55] E. Ardonne, N. Read, E. Rezayi, and K. Schoutens, Nucl. Phys. B 607, 549 (2001).

[56] C.-C. Chang, N. Regnault, T. Jolicoeur, and J. K. Jain, Phys. Rev. A 72, 013611 (2005).

[57] X. G. Wen, Int. J. Mod. Phys. B6, 1711 (1992).

[58] M. A. Cazalilla, Phys. Rev. A 67, 063613 (2003).

[59] B. Juliá-Díaz and T. Graß, Comput. Phys. Commun. 183, 737 (2012).

[60] J. Jain, Composite Fermions (Cambridge University Press, Cambridge, UK, 2007).

[61] N. R. Cooper, E. H. Rezayi, and S. H. Simon, Phys. Rev. Lett. 95, 200402 (2005).

[62] D. Green, Ph.D. thesis, Yale University, 2001. 